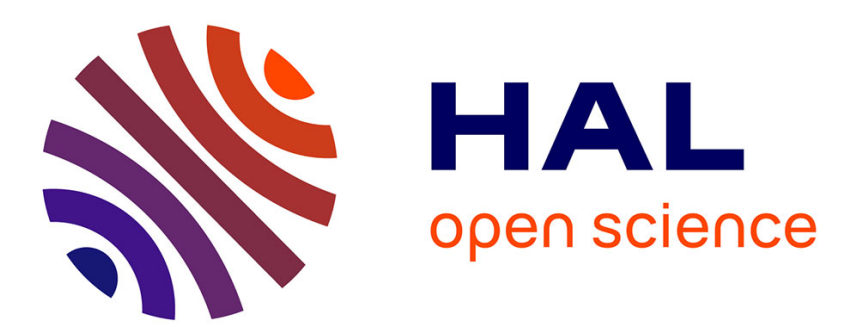

\title{
Closed-form Coverage Probability under the Idle Mode Capability: A Stochastic Geometry Approach
}

\author{
Yassine Hmamouche, Mustapha Benjillali, Samir Saoudi
}

\section{To cite this version:}

Yassine Hmamouche, Mustapha Benjillali, Samir Saoudi. Closed-form Coverage Probability under the Idle Mode Capability: A Stochastic Geometry Approach. ISIVC 2018: 9th International Symposium on Signal Image Video and Communications, Nov 2018, Rabat, Morocco. 10.1109/ISIVC.2018.8709219 . hal-01893752

HAL Id: hal-01893752

https://hal.science/hal-01893752

Submitted on 11 Oct 2018

HAL is a multi-disciplinary open access archive for the deposit and dissemination of scientific research documents, whether they are published or not. The documents may come from teaching and research institutions in France or abroad, or from public or private research centers.
L'archive ouverte pluridisciplinaire HAL, est destinée au dépôt et à la diffusion de documents scientifiques de niveau recherche, publiés ou non, émanant des établissements d'enseignement et de recherche français ou étrangers, des laboratoires publics ou privés. 


\title{
Closed-form Coverage Probability under the Idle Mode Capability: A Stochastic Geometry Approach
}

\author{
Yassine Hmamouche ${ }^{1,2}$, Mustapha Benjillali ${ }^{2}$, and Samir Saoudi ${ }^{1}$ \\ ${ }^{1}$ IMT Atlantique, Lab-STICC, UBL, 29238 Brest, France \\ Emails: \{yassine.hmamouche; samir.saoudi\}@imt-atlantique.fr \\ ${ }^{2}$ Communication Systems Department, INPT, Rabat, Morocco \\ Email: benjillali@ieee.org
}

\begin{abstract}
In this paper, we provide a comprehensive overview of the stochastic geometry framework explored extensively in literature to model and analyze the performance of communication networks. We provide a brief survey of the history of the approach, including recent applications to the analysis of several 5G enabling technologies. In the main technical section of the paper, we consider a case study in which we assume a system model where the idle mode capability is activated on base stations. A general expression of the coverage probability based on hypergeometric functions is then derived, yielding closedform expressions that depend on the parity of the path loss exponent. Numerical results confirm the accuracy of our practical approximations.
\end{abstract}

\section{INTRODUCTION}

Enhanced capacity, improved service quality, lower power consumption, and ubiquitous coverage, are some of the most relevant objectives for the deployment of recent communication networks. The first step towards achieving these targets is an efficient approach to the modeling of these networks. In earlier years, modeling methods were mainly based on detailed geographical descriptions of the network; the accuracy of future results depended on the extrapolation of the actual state of the network for some evolution scenarios. To meet the expected demand, strategic planning must decide between a few potential architectures where costs and performance are assessed through detailed calculations. This approach, still adopted nowadays, generally requires the development of specific optimization softwares adapted to each network architecture, and relies on heavy, time-consuming, simulations [1], [2].

To address these limitations, tools from applied probability, particularly stochastic geometry (SG) and point process theory, have been introduced to model and analyze the communication networks [2]-[4]. SG provides a natural way to define and derive macroscopic properties for such networks, by averaging over all potential geometric models for the active nodes (mobile users, base stations in a cellular network, access points in a WiFi network, switching centers [5], ...), which often

This work is funded by a research grant from PRACOM and the Regional Council of Brittany, France. capture the major dependencies between the network performance characteristics (connectivity, stability, capacity, ...) as a function of a relatively small number of parameters. In fact, a communications network can be viewed as a set of nodes, where locations can be seen as the realization of a point process in a given domain $\mathbb{R}^{d}$ [6]. At any given time, the signal received from the serving transmitter may be affected by the signals received from the other transmitters. The geometry of the locations of the nodes then plays a key role since it determines the signal-to-interference-plus-noise ratio (SINR) at each receiver and, subsequently, assesses the possibility of simultaneously establishing any collection of links at a given bit rate.

The seminal work in [2] provides a comprehensive understanding about the behavior of downlink cellular networks. By assuming that single-antenna base stations (BSs) are distributed according to a homogeneous Poisson point process (PPP), a cell association criterion based on the highest average received power, a single-slope path-loss model, and Rayleigh fading; the authors have obtained a tractable integral expression of the coverage probability. An important outcome is the SINR invariance property, which states that the SINR increases almost linearly with BS density to the point where noise becomes negligible; after which SINR remains stable and independent from the BS density. Another important outcome is a closed-form expression developed for the integral expression when the path loss exponent is equal to 4 .

The motivation behind this paper is then to develop a closed-form expression for the coverage probability, which will encompass the expression in [2], and will be valid for all integer values of the path loss exponent. To do so, we explore the potential of hypergeometric functions analysis and consider a more general system model in which the idle mode capability (IMC) is activated on BSs.

The remainder of the paper is organized as follows. Section II introduces the contextual background of SG as a powerful tool for modeling communications networks and analyzing the performance of several 5 th generation $(5 \mathrm{G})$ enabling technologies. Section III is the technical part of the paper, 
in which we develop a general expression for the coverage probability that yields closed-form expressions depending on the parity of the path loss exponent. In Section IV, numerical results are reported to corroborate our theoretical findings and assess the accuracy of our approximations, and Section V concludes the paper.

\section{Stochastic Geometry BASEd ApproAch}

In this section, we discuss basic insights to better understand the context of the SG approach. We briefly present the history of SG, address the fundamental concepts of modeling communication networks using SG, and summarize the state of the art of SG-based analysis for several 5G enabling technologies.

In fact, SG is an old branch of probability theory that deals with random geometrical structures such as point processes, random graphs, and random networks, as well as the challenging problems raised by their statistical analysis. The subject has progressed rapidly in response to the challenges from different applied sciences, from image analysis to communication networks.

In telecommunications, SG models are designed to capture aspects of wireless networks such as the association policy between users and nodes, interference characterization and interaction between nodes and/or users (repulsion, attraction, or zero interaction). The related research consists in analyzing these models with the aim of better predicting and controlling various network performance metrics.

\section{A. Brief Historical Review}

SG as a concept of geometric probability is a field with roots going back at least 300 years. In fact, the connection between probability theory and geometry can be traced back at least to the middle of the 18th century when Buffon's needle problem (1733), and subsequently questions related to Sylvester's four point problem (1864) and Bertrand's paradox (1889), started to challenge prominent mathematicians, and helped advance the probabilistic modeling. A short historical outline of these early days of geometric probability may be found in [7], [8].

Since the 1950s, the framework of geometric probability broadened substantially and has been framed as an academic area. In particular, the focus mainly switched to models involving a typical number of randomly selected geometric objects. As a consequence, the notion of point processes started to play a prominent role in this field, which since then was called "stochastic geometry". Simultaneously, relevant models were developed for wireless network technologies including mobile ad hoc networks, sensor networks, vehicular networks, and cognitive radio (CR) networks [9], [10].

Around the beginning of the 21 st century, a number of new network technologies have arisen such as heterogeneous (HetNet) and millimeter wave cellular networks. SG has been extensively explored to develop models for these networks [2], [3] and then derive closed-form or semi-closed-form expressions for performance metrics and quality of service (QoS) indicators, without resorting to simulation methods or intractable deterministic models. The aforementioned metrics and indicators are often based on concepts from information theory such as the SINR; which forms the mathematical basis for defining network connectivity and coverage.

\section{B. Stochastic Geometry and the Modeling of Communication Networks}

The contemporary network systems have a complicated multi-layer and multi-level architecture. Consequently, the network performance investigated for purposes of strategic planning and economic analysis is dependent on a large number of parameters, where it is generally difficult to determine in advance which are the main that mostly influence the desirable properties of the system. On the other hand, exhaustive simulations are time and resource consuming, and do not offer the ability to formally determine the structural parameters impacting the system.

SG resolves the aforementioned challenges by providing macroscopic network models that capture the essential spatial characteristics of networks performance and then economy through a lower number of structural parameters [1]. This way, the production function of telecommunication services can be analytically expressed where the main players in the network (subscribers/users, stations, links, mobiles) are considered as a realization of a family of stochastic processes belonging to simple parametric classes. Fig. 1 describes the typical architecture of a SG based model [1].

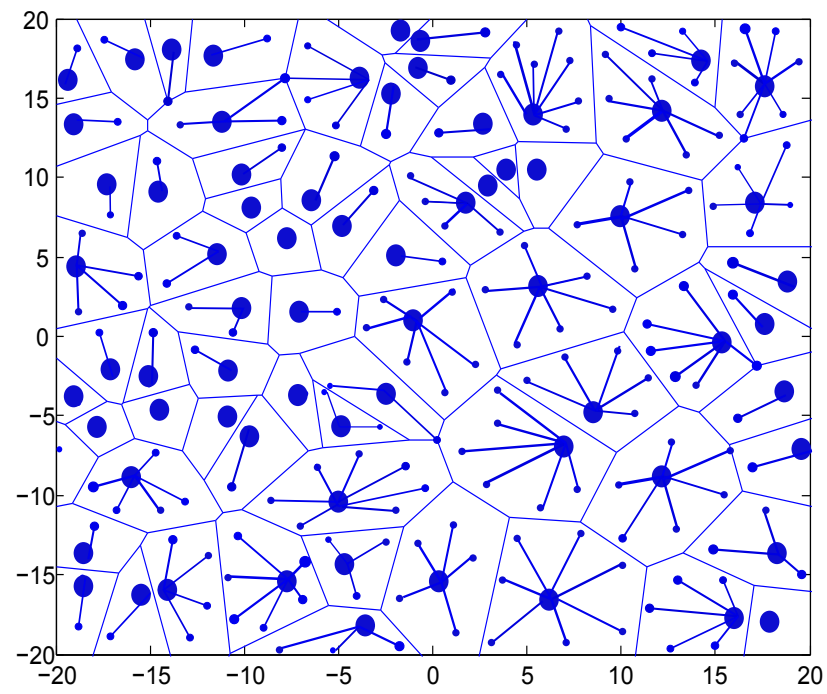

Fig. 1. A typical configuration of a stochastic geometry based model, where the distribution of users and nodes follows homogenous PPPs. The connections between nodes and users are shown in a single hierarchical level, and the cells boundaries form a Voronoi tessellation.

\section{Stochastic Geometry and 5G Technologies}

The $5 \mathrm{G}$ mobile communication networks are expected to be operational by 2020, many efforts and revolutionary ideas have been proposed and explored around the world to satisfy the increasing demand for higher data rates, lower network 
latencies, better energy efficiency, and reliable ubiquitous connectivity. The primary technologies and approaches to address the requirements of $5 \mathrm{G}$ systems include:

- the densification of existing cellular networks with massive addition of small cells;

- peer-to-peer (P2P) communications enabling multinetwork association (e.g., device-to-device (D2D) and machine-to-machine (M2M) communication enabled multi-tier HetNets);

- simultaneous transmission and reception (e.g., full-duplex (FD) communications);

- massive multiple-input multiple-output (massive MIMO);

- millimeter wave communication technologies;

- improved energy efficiency via energy-aware communication/design and energy harvesting;

- and new radio access techniques (e.g., non-orthogonal multiple access (NOMA)).

Fig. 2 describes the main components of a typical $5 \mathrm{G}$ network architecture.

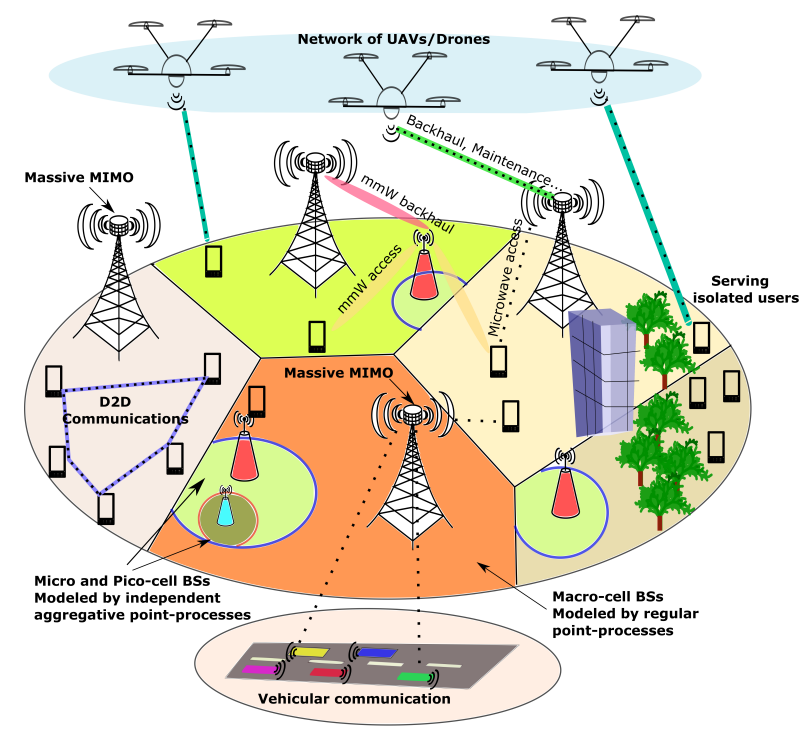

Fig. 2. A typical architecture of a $5 \mathrm{G}$ network where small cells are modeled by aggregative point processes and macro cells are modeled by regular point processes.

Exploiting the power of SG, several notable results are obtained for cellular networks. For instance, the authors of [11] investigated the performance behavior of ultra-dense networks and confirmed that the SINR invariance property is no longer valid as the dual-slope path loss model is considered and the near-field path loss exponent is under the dimension of the network. Moreover, authors of [12], [13] revealed that antenna heights and omni-directionality are so detrimental to the network performance during ultra-densification. However, It is proved in [14] and references therein that the coverage probability has non-zero convergence in asymptotic regions of ultra-densification unless UE density grows unbounded.

On the other side, as the density of small cells increase is limited by a potential constraint on backhaul capacity, downloading contents from the core network via backhaul links becomes the bottleneck of the system, impairing the throughput gain brought by the network densification. To overcome this, an innovative approach is considered where new cells, so-called helper nodes, are introduced into the network. These nodes have typically weak backhaul links and high capacity caches, so the content is retrieved locally instead of fetching it from the core network via backhaul links. Therefore, the backhaul link is used only to refresh the caches at off-peak times. Authors of [15] investigated the performance gain of a cache-enabled HetNet over a conventional HetNet with limited capacity backhaul.

The Massive MIMO technique exploring especially high number of antennas is characterized in [4], [16]. Since energy efficiency (EE) not only has great ecological benefits and represents social responsibility, but also has significant economic benefits, the authors of [17] apply tools from SG to analyze the energy efficiency of cellular networks through the use of the sleeping mode capability of BSs where the active or sleep mode of a BS is activated based on the cell load. In [18], the authors considered the energy harvesting where the system model consists of HetNet classes of self-powered BSs, which may differ in terms of the transmit power, deployment density, energy harvesting rate, and energy storage capacity. The objective was to characterize the regimes in which the self-powered network may have the same performance as the one with reliable energy sources. Extensions to the radio frequency $(\mathrm{RF})$ energy harvesting is considered in the analysis in [19].

Based on dynamic spectrum access and spectrum sharing, CR technology is analyzed in [20] where a SG based analysis is considered for a combination of cognitive, energy harvesting and D2D based cellular network.

Recently, NOMA have received significant attention for the design of $5 \mathrm{G}$ radio access techniques. Unlike with the traditional orthogonal multiple access (OMA) techniques, where different users are served over orthogonal resource blocks, NOMA allows multiple users to share the same resource block. Based on SG, the baseline of a power-domain NOMA is investigated in [21].

From another point of view, securing communications is a critical issue for future $5 \mathrm{G}$ networks where there will be a great need for new wireless security mechanisms that do not necessarily rely on the usual bit-level protocols. Thus, based on the SG tractability, the physical layer security in the context of cellular networks and mmWave channel characteristics is discussed in [22].

\section{Coverage ANAlysis}

In this section, we consider a downlink cellular network in which BSs and users are scattered according to two independent homogeneous PPPs $\Psi$ and $\Psi_{u}$ respectively with respective densities $\lambda$ and $\lambda_{u}$. We assume that IMC is activated, then since users density is finite, ultra-densification will lead that some BSs will remain switched on with no users being served, hence these BSs may be put in idle state in order to mitigate the inter-cell interference and reduce the energy consumption. Based on the legacy of hypergeometric functions 
analysis, we aim to develop a closed-form expression of the coverage probability in [2, Th. 2] by encompassing the $\mathcal{Q}$ function based expression valid only for a path-loss exponent $\alpha=4$.

We define the coverage probability $p_{\mathrm{c}}$ as the probability that the long-term SINR, is greater that a threshold $T$. It is equivalently the CCDF of SINR over the entire network and can be considered [2] as (i) the probability that a randomly selected user can reach a target $\mathrm{T}$, (ii) the average fraction of users who reach $\mathrm{T}$ at any given time, (iii) the average fraction of the network area covered at any given time. Similarly, the concept of outage probability $p_{\mathrm{o}}=1-p_{\mathrm{c}}$ (CDF of SINR), is conceived as the probability of not being able to send a signal or symbol over a specific channel, and thus ${ }^{1}$

$$
\begin{gathered}
p_{\mathrm{c}}(\mathrm{T})=1-p_{\mathrm{o}}(\mathrm{T})=\mathbb{P}\{\mathrm{SINR} \geq T\}, \\
\text { where } \\
\operatorname{SINR}=\frac{h l\left(r_{0}\right)}{\mathrm{I}_{\mathrm{agg}}+\sigma^{2}},
\end{gathered}
$$

$h$ is the fading power of the channel between the typical user and the serving $\mathrm{BS}\left\{b_{0}\right\}, r_{0}$ is the distance between the typical user and the serving BS assuming the nearest BS association policy, $l(r)=r^{-\alpha}$ is the path loss function such as $\alpha$ is the path loss exponent, $\sigma^{2}$ is the noise variance normalized by the BS transmit power $\mathrm{P}_{\mathrm{tx}}$, and $\mathrm{I}_{\mathrm{agg}}=\sum_{y_{i} \in \Psi \backslash\left\{b_{0}\right\}} g_{i} l\left(y_{i}\right)$ is the other-cell interference such as $g_{i}$ is the fading power of the channels between the typical user and the interfering BSs.

We focus on the expression of $p_{\mathrm{c}}$ as the calculation of almost all the other metrics requires the preliminary calculation of $p_{\mathrm{c}}$. In fact, the average ergodic rate $\tau$ may be seen as

$$
\begin{aligned}
\tau & \triangleq \mathbb{E}\{\ln (1+\mathrm{SINR})\} \\
& \stackrel{(\mathrm{a})}{=} \int_{t>0} \mathbb{P}\{\ln (1+\mathrm{SINR})>t\} \mathrm{d} t \\
& =\int_{t>0} \mathbb{P}\left\{\mathrm{SINR}>e^{t}-1\right\} \mathrm{d} t=\int_{t>0} p_{\mathrm{c}}\left(e^{t}-1\right) \mathrm{d} t \\
& =\int_{u>0} \frac{p_{\mathrm{c}}(u)}{u+1} \mathrm{~d} u \stackrel{(\mathrm{b})}{=}-\int_{u>0} \ln (1+u) \frac{\partial p_{\mathrm{c}}(u)}{\partial u} \mathrm{~d} u,
\end{aligned}
$$

where (a) follows from the property $\mathbb{E}\{X\}=$ $\int_{t>0} \mathbb{P}\{X>t\} \mathrm{d} t$ for a positive random variable $X$ and (b) follows by applying integration by parts where $\lim _{u \rightarrow 0} p_{\mathrm{c}}(u)=1$ and $\lim _{u \rightarrow \infty} p_{\mathrm{c}}(u)=0$.

Moreover, in practical systems where a minimum operational SINR, namely $\gamma_{0}$, is required, the constrained area spectral efficiency $\mathrm{ASE}_{\mathrm{c}}$ is expressed as ${ }^{2}$

$$
\begin{aligned}
\mathrm{ASE}_{\mathrm{c}} & =\lambda \mathbb{E}\{\ln (1+\mathrm{SINR})\} \mathbb{1}\left(\operatorname{SINR} \geq \gamma_{0}\right) \\
& =\lambda \int_{\gamma_{0}}^{\infty} \ln (1+\gamma) f_{\Gamma}(\gamma) \mathrm{d} \gamma \\
& =\lambda\left[\int_{\gamma_{0}}^{\infty} \frac{p_{\mathrm{c}}(\gamma)}{1+\gamma} \mathrm{d} \gamma+\ln \left(1+\gamma_{0}\right) p_{\mathrm{c}}\left(\gamma_{0}\right)\right],
\end{aligned}
$$

\footnotetext{
${ }^{1} \mathbb{P}(X)$ stands for the probability of the event $X$ and $\mathbb{E}(X)$ for the expected value of the event $X$.

${ }^{2}$ The spectral efficiency is measured in nats $/ \mathrm{s} / \mathrm{Hz}$, and need to be normalized by $\ln (2)$ to translate into $\mathrm{bit} / \mathrm{s} / \mathrm{Hz}$.
}

where $f_{\Gamma}($.$) is the PDF of SINR observed at the typical user,$ and expressed as

$$
f_{\Gamma}(\gamma)=\frac{\partial\left(1-p_{\mathrm{c}}(\gamma)\right)}{\partial \gamma}
$$

Based on some standard assumptions, i.e. singular path-loss model, Rayleigh fading, BSs and users equipped with single antennas, and a cell association policy based on the highest average received power, the authors of [2] have obtained the following tractable expression of $p_{\mathrm{c}}$

$$
p_{\mathrm{c}}(\mathrm{T})=\pi \lambda \int_{0}^{\infty} e^{-a u^{\alpha / 2}-b u} \mathrm{~d} u
$$

where $a=\mathrm{T} \sigma^{2}$ captures noise, $b=$ $\pi \lambda\left[1-(1-p)\left(1-{ }_{2} F_{1}(1,-\delta ; 1-\delta ;-\mathrm{T})\right)\right] \quad$ reflects interference such as $\delta=2 / \alpha,{ }_{2} F_{1}(., . ; . ; z)$ is the Gauss hypegeometric function and $p$ is the empty-cell probability, i.e, the probability that a typical user is associated to no mobile. $p$ is generally approximated by averaging the void probability of $\Psi_{u}$ over the distribution of a typical Voronoi cell area. Assuming a network deployed in the 2-D plane $\mathbb{R}^{2}$, the authors of [5] approximated $p$ as

$$
p \simeq\left(1+\frac{\lambda_{u}}{3.5 \lambda}\right)^{-3.5}
$$

Generally, the coverage probability in (5), cannot be formulated in closed-form expression. An exception is made when considering a path loss exponent of $\alpha=4$ or assuming the interference-limited regime [2, Formulas (7) and (8)]. The following theorem develop a closed-form expression for $p_{\mathrm{c}}$ that is valid for every integer path loss exponent $\geq 3$.

Theorem 1. For integer path-loss exponents $\alpha \geq 3$, the coverage probability in (5) will be expressed in closed-form as

$$
\begin{aligned}
p_{c}(\mathrm{~T})=\frac{2 \pi \lambda}{\alpha\left(\mathrm{T} \sigma^{2}\right)^{\frac{2}{\alpha}}} & \sum_{k=0}^{\frac{\alpha}{2}-1} \frac{(-1)^{k} \kappa^{k}}{k !} \Gamma\left(\frac{2+2 k}{\alpha}\right) \\
& \times{ }_{1} F_{\frac{\alpha-2}{2}}\left(\frac{4+2 k}{\alpha}, \ldots, \frac{\alpha+2 k}{\alpha} \mid \frac{(-\kappa)^{\frac{\alpha}{2}}}{\left(\frac{\alpha}{2}\right)^{\frac{\alpha}{2}}}\right),
\end{aligned}
$$

for $\alpha$ even, and as,

$$
\begin{aligned}
p_{c}(\mathrm{~T})=\frac{2 \pi \lambda}{\alpha\left(\mathrm{T} \sigma^{2}\right)^{\frac{2}{\alpha}}} & \sum_{k=0}^{\alpha-1} \frac{(-1)^{k} \kappa^{k}}{k !} \Gamma\left(\frac{2+2 k}{\alpha}\right) \\
& \times{ }_{2} F_{\alpha-1}\left(\begin{array}{c}
1, \frac{1}{2}+\frac{k+1}{\alpha} \\
\frac{2+k}{\alpha}, \ldots, \frac{\alpha+k}{\alpha} \mid
\end{array} \mid \frac{4(-\kappa)^{\alpha}}{\alpha^{\alpha}}\right),
\end{aligned}
$$

for $\alpha$ odd, where

$$
\kappa=\frac{1}{\left(T \sigma^{2}\right)^{2 / \alpha}} \pi \lambda\left[1-(1-p)\left(1-{ }_{2} F_{1}(1,-\delta ; 1-\delta ;-\mathrm{T})\right)\right] .
$$

and ${ }_{p} F_{q}($.$) is the generalized hypergeometric function, defined$ for complex parameters and argument by the series

$$
{ }_{p} F_{q}\left(\begin{array}{c}
a_{1}, a_{2}, \ldots, a_{p} \\
b_{1}, b_{2}, \ldots, b_{q}
\end{array} \mid z\right)=\sum_{k=0}^{\infty} \frac{\left(a_{1}\right)_{k}\left(a_{2}\right)_{k} \cdots\left(a_{p}\right)_{k}}{\left(b_{1}\right)_{k}\left(b_{2}\right)_{k} \cdots\left(b_{q}\right)_{k}} \frac{z^{k}}{k !},
$$


where $(a)_{k}$ is the Pochhammer symbol.

Proof. By the variable change of $x=a u^{\alpha / 2}$, the expression (5) of coverage probability can be rewritten as

$$
\begin{aligned}
p_{\mathrm{c}}(T) & =\frac{2 \pi \lambda}{\alpha a^{\alpha / 2}} \int_{0}^{\infty} x^{\frac{2}{\alpha}-1} e^{-x} e^{-\kappa x^{2 / \alpha}} \mathrm{d} x \\
& =\frac{2 \pi \lambda}{\alpha a^{\alpha / 2}} \int_{0}^{\infty} x^{\frac{2}{\alpha}-1} e^{-x}{ }_{0} F_{0}\left(. ; ;-\kappa x^{2 / \alpha}\right) \mathrm{d} x .
\end{aligned}
$$

Depending on the parity of $\alpha$, and using [23, Eq. (43)] which states for any natural number $m$ that

$$
\begin{aligned}
& { }_{p} F_{q}\left(\begin{array}{c}
a_{1}, a_{2}, \ldots, a_{p} \\
b_{1}, b_{2}, \ldots, b_{q}
\end{array} \mid z\right)=\sum_{k=0}^{m-1} \frac{\left(a_{2}\right)_{k}\left(a_{2}\right)_{k} \ldots\left(a_{p}\right)_{k}}{\left(b_{1}\right)_{k}\left(b_{2}\right)_{k} \ldots\left(b_{p}\right)_{k}} \frac{z^{k}}{k !} \\
& { }_{m p+1} F_{m q+m}\left(\underset{B_{1, k}}{\stackrel{\overrightarrow{A_{1, k}}}{\longrightarrow}, \overrightarrow{B_{2, k}}, \ldots, \overrightarrow{A_{2, k}}, \ldots, \overrightarrow{B_{q, k}}, \overrightarrow{I_{1, k}}} \mid m^{(p-q-1) m} z^{m}\right) \text {, }
\end{aligned}
$$

where for $j=1,2, \cdots, p$ and $i=1,2, \cdots, q$

$$
\begin{aligned}
& \overrightarrow{A_{j, k}}=\left(\frac{a_{j}+k}{m}, \frac{a_{j}+k+1}{m}, \ldots, \frac{a_{j}+m-1+k}{m}\right) \\
& \overrightarrow{B_{i, k}}=\left(\frac{b_{i}+k}{m}, \frac{b_{i}+k+1}{m}, \ldots, \frac{b_{i}+m-1+k}{m}\right)
\end{aligned}
$$

and

$$
\overrightarrow{I_{1, k}}=\left(\frac{1+k}{m}, \frac{2+k}{m}, \ldots, \frac{m+k}{m}\right)
$$

Thus, for the even case, we choose $m=\alpha / 2$, while for the odd case, we choose $m=\alpha$. Using the integral transformation of hypergeometric functions in $[24,(1.7 .525)]$, we get the desired result.

Note that the expression in [2, Th. 2] can be obtained directly from (7) where the Gaussian $Q$-function is expressed as

$$
Q(x)=\frac{e^{-\frac{x^{2}}{2}}}{2}\left[{ }_{0} F_{0}\left(-;-; \frac{x^{2}}{2}\right)-\frac{x \sqrt{2}}{\sqrt{\pi}}{ }_{1} F_{1}\left(1 ; \frac{3}{2} ; \frac{x^{2}}{2}\right)\right] .
$$

The accuracy of the analysis is discussed in the following section.

\section{Numerical Results}

In Fig. 3, the coverage probability grows monotonically with the path loss exponent where the results of the expression from Theorem 1 are matching perfectly with the integration in (5).

In Fig. 4, the coverage probability decreases as the users process density $\lambda_{u}$ increases, this may be explained intuitively by the observation that the average number of interfering BSs increases with users density due to the IMC activation. Moreover, it is interesting to mention that the SINR target T reduces the impact of users process density $\lambda_{u}$ on the SINR CCDF. In fact, a lower $\mathrm{T}$ is generally common in the noiselimited regime where few BSs are deployed and therefore reduced impact of IMC activation (e.g., rural environments).

Fig. 5 shows the scaling of coverage probability with users density $\lambda_{u}$ considering different values of BSs density $\lambda$ and three formulations of the empty-cell probability $p$. The first

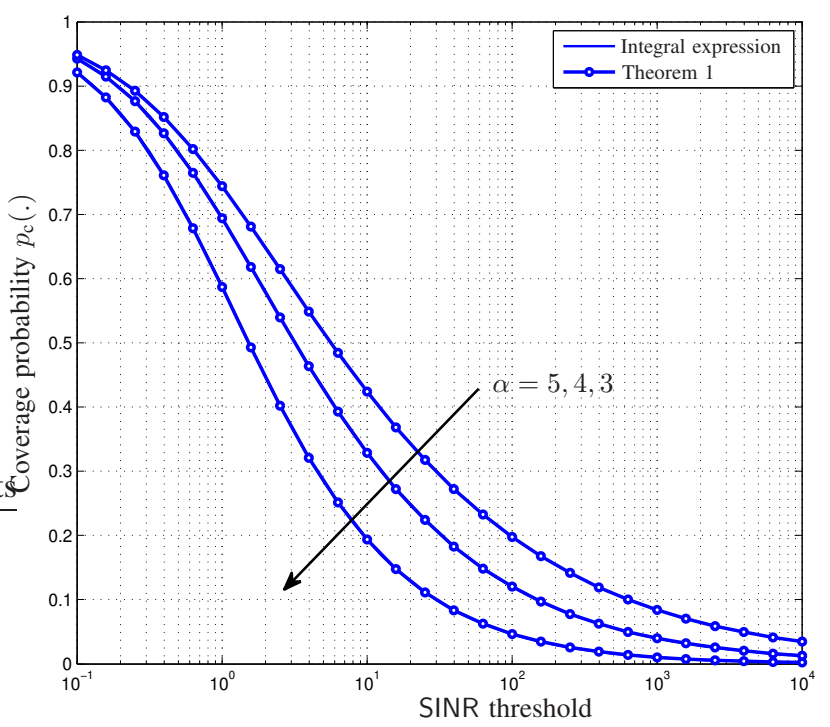

Fig. 3. The scaling of coverage probability with the path loss exponent $\alpha$, the hypergeometric formulation of Theorem 1 is very tight with the integral expression where $\lambda=0.7, \lambda_{u}=0.2, \sigma^{2}=1$.

one is that of (6), the second is by considering the case of ultradense networks where $\lambda \gg \lambda_{u}$, in other words, a considerable increase in $\lambda_{u}$ generates a corresponding increase in $\lambda$, and thus $p$ may be approximated as

$$
p=\left(1+\frac{\lambda_{u}}{3.5 \lambda}\right)^{-3.5} \simeq 1-\frac{\lambda_{u}}{\lambda} .
$$

Moreover, (10) may be seen as the first terms of an exponential Taylor expansion. Thus, we consider a third approximation of $p$ in a more tractable way as $p \simeq e^{-\frac{\lambda_{u}}{\lambda}}$. The process of the transmitting BSs can then be approximated as a thinning transformation of density $\lambda e^{-\frac{\lambda u}{\lambda}}$.

Note that the exponential approximation captures perfectly the trends of coverage probability under (6). In fact, $p$ goes towards 0 as $\lambda_{u}$ increases, i.e. BSs continually transmit signals, and the tagged user receives interference from all BSs, and then the coverage probability becomes independent from $\lambda_{u}$. However, under the second approximation (10), the coverage probability remains dependent on users density and goes towards 0 as $\lambda_{u}$ increases.

\section{CONCLUSION}

The field of SG is rapidly evolving in conjunction with numerous ongoing developments from a variety of applications, and offers an interesting framework for the analysis of communication networks.

In this paper, we reviewed the context of SG based approaches and their application to the analysis of new $5 \mathrm{G}$ technologies. Moreover, the coverage probability under the IMC mode is investigated, and closed-form approximations are developed.

Our numerical results confirm that the hypergeometric expressions are perfectly matching with the numerical integration 


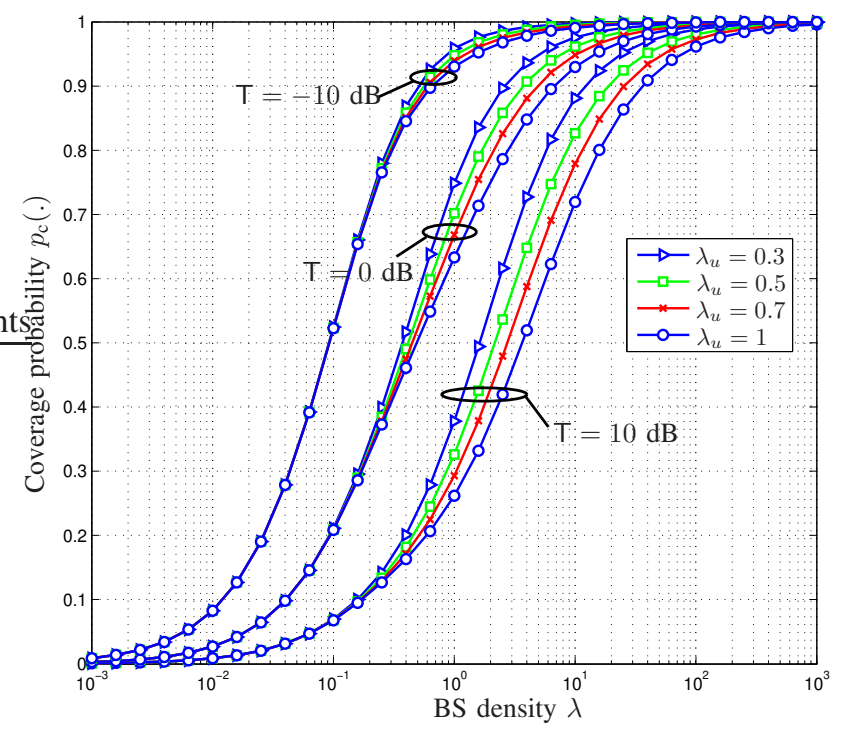

Fig. 4. The scaling of coverage probability with the BS density $\lambda$ for $\alpha=4$ and different values of users density $\lambda_{u}$ and SINR thresholds T.

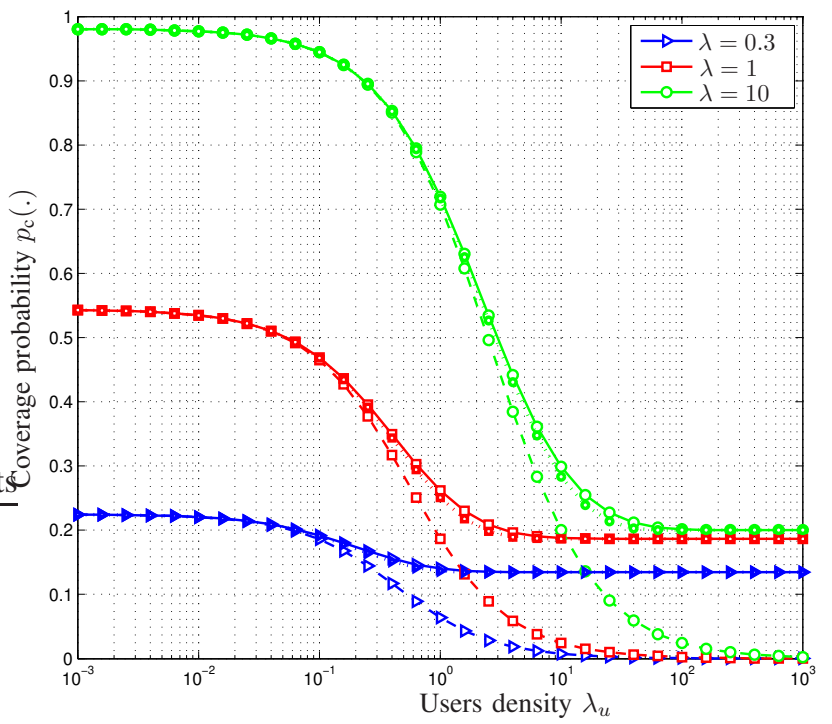

Fig. 5. The scaling of coverage probability with the users density $\lambda_{u}$ for different values of BS density $\lambda$ and the empty-cell probability $p$. the solid line is for $p=\left(1+\left(\lambda_{u} / 3.5 \lambda\right)\right)^{-3.5}$, the dashed line is for the approximation of $p$ by $\exp \left(-\lambda_{u} / \lambda\right)$, and the dotted line is for the approximation of $p$ by $1-\left(\lambda_{u} / \lambda\right)$.

when the path loss exponent is of integer value. The exponential approximation of the empty-cell probability is tight enough; particularly for low users densities.

\section{REFERENCES}

[1] F. Baccelli, M. Klein, M. Lebourges, and S. Zuyev, "Stochastic geometry and architecture of communication networks," Select Proceedings of the third INFORMS telecommunications conference, 1996.

[2] J. G. Andrews, F. Baccelli, and R. K. Ganti, "A tractable approach to coverage and rate in cellular networks," IEEE Trans. Commun., vol. 59, no. 11, pp. 3122-3134, Nov. 2011.

[3] H.-S. Jo, Y. J. Sang, P. Xia, and J. G. Andrews, "Heterogeneous cellular networks with flexible cell association: A comprehensive downlink SINR analysis," IEEE Trans. Wireless Commun., vol. 11, no. 10, pp. 34843495, Oct. 2012.
[4] M. Di Renzo and P. Guan, "A Mathematical Framework to the Computation of the Error Probability of Downlink MIMO Cellular Networks by Using Stochastic Geometry," in IEEE Transactions on Communications, vol. 62, no. 8, pp. 2860-2879, Aug. 2014.

[5] S. Lee and K. Huang, "Coverage and economy of cellular networks with many base stations," IEEE Communications Letters, vol. 16, no. 7, pp. 1038-1040, Jul. 2012.

[6] F. Baccelli and B. Blaszczyszyn, "Stochastic Geometry and Wireless Networks, Volume I-Theory," Hanover, MA, USA: Now, 2009.

[7] R. Schneider and W. Weil, "Stochastic and integral geometry," Probability and its Applications (New York), Springer-Verlag, Berlin, 2008.

[8] R. Schneider and W. Weil, "Classical stochastic geometry," Kendall W.S and Molchanov I. (eds.): New perspectives in stochastic geometry, pp. $1-42,2010$.

[9] L. Kleinrock and J. Silvester, "Optimum transmission radii for packet radio networks or why six is a magic number," In IEEE National Telecommunications, pp. 4.31-4.35, 1978.

[10] H. Takagi and L. Kleinrock, "Optimal transmission ranges for randomly distributed packet radio terminals," In IEEE Transactions on Communications, pp. 246-257, 1984.

[11] A. K. Gupta, X. Zhang and J. G. Andrews, "SINR and Throughput Scaling in Ultradense Urban Cellular Networks," in IEEE Wireless Communications Letters, vol. 4, no. 6, pp. 605-608, Dec. 2015.

[12] I. Atzeni, J. Arnau and M. Kountouris, "Downlink cellular network analysis with LOS/NLOS propagation and elevated base stations, in IEEE Transactions on Wireless Communications, vol. 17, no. 1, pp. 142-156, Jan. 2018.

[13] A. Merwaday, R. Vannithamby, M. M. Rashid, Y. Zhang, C. Chen and X. $\mathrm{Wu}$, "On the performance of directional communications in ultra-dense networks, 2017 IEEE International Conference on Communications Workshops (ICC Workshops), Paris, 2017, pp. 522-527.

[14] Y. Yang, J. Park and K. W. Sung, "On the Asymptotic Behavior of UltraDensification under a Bounded Dual-Slope Path Loss Model," European Wireless 2017; 23th European Wireless Conference, Dresden, Germany, 2017, pp. 1-7.

[15] D. Liu and C. Yang, "Cache-enabled heterogeneous cellular networks: Comparison and tradeoffs," 2016 IEEE International Conference on Communications (ICC), Kuala Lumpur, 2016, pp. 1-6.

[16] H. S. Dhillon, M. Kountouris and J. Andrews, "Downlink MIMO HetNets: Modeling, Ordering Results and Performance Analysis," IEEE Trans. Wireless Commun., vol. 12, no. 10, pp. 52085222, Oct. 2013.

[17] Y. S. Soh, T. Q. S. Quek, M. Kountouris and H. Shin, "Energy Efficient Heterogeneous Cellular Networks," in IEEE Journal on Selected Areas in Communications, vol. 31, no. 5, pp. 840-850, May 2013.

[18] H. S. Dhillon, Y. Li, P. Nuggehalli, Z. Pi and J. G. Andrews, "Fundamentals of Heterogeneous Cellular Networks with Energy Harvesting," in IEEE Transactions on Wireless Communications, vol. 13, no. 5, pp. 2782-2797, May 2014.

[19] I. Flint, X. Lu, N. Privault, D. Niyato and P. Wang, "Performance Analysis of Ambient RF Energy Harvesting with Repulsive Point Process Modeling," in IEEE Transactions on Wireless Communications, vol. 14, no. 10, pp. 5402-5416, Oct. 2015.

[20] A. H. Sakr and E. Hossain, "Cognitive and Energy Harvesting-Based D2D Communication in Cellular Networks: Stochastic Geometry Modeling and Analysis," in IEEE Transactions on Communications, vol. 63, no. 5, pp. 1867-1880, May 2015.

[21] Z. Zhang, H. Sun and R. Q. Hu, "Downlink and Uplink Non-Orthogonal Multiple Access in a Dense Wireless Network," in IEEE Journal on Selected Areas in Communications, vol. 35, no. 12, pp. 2771-2784, Dec. 2017.

[22] C. Wang and H. M. Wang, "Physical Layer Security in Millimeter Wave Cellular Networks," in IEEE Transactions on Wireless Communications, vol. 15 , no. 8, pp. 5569-5585, Aug. 2016.

[23] A. P. Prundnikov, Y. A. Brychkov and O. I. Marichev, Integrals and Series, Volume 3: More Special Functions. Gordon and Breach Science Publishers, Philadelphia (1990).

[24] I. S. Gradshteyn,I. M. Ryzhik: Table of Integrals, Series, and Products, 7th edn. Academic Press, Elsevier Inc (2007) 